\title{
Fertilidade do solo e estado nutricional do cafeeiro cultivado em atmosfera enriquecida com $\mathrm{CO}_{2}$
}

\author{
Henrique José Guimarães Moreira Maluf(1), Raquel Ghini(2), Laura Beatriz Batista de Melo(1) \\ e Carlos Alberto Silva ${ }^{(1)}$
}

\begin{abstract}
(1)Universidade Federal de Lavras, Departamento de Ciência do Solo, Campus Universitário, Caixa Postal 3037, CEP 37200-000 Lavras, MG, Brasil. E-mail: maluf.henrique@yahoo.com.br, lauramelo26@hotmail.com, csilva@dcs.ufla.br (2)Embrapa Meio Ambiente, Rodovia SP-340, Km 127,5, Tanquinho Velho, CEP 13820-000 Jaguariúna, SP, Brasil. E-mail: raquel.ghini@embrapa.br
\end{abstract}

Resumo - O objetivo deste trabalho foi avaliar os efeitos de concentrações de $\mathrm{CO}_{2}$ atmosférico nos atributos químicos do solo, na linha (cafeeiro) e na entrelinha (braquiária), e nos teores de macronutrientes em folhas do cafeeiro. Utilizou-se o delineamento de blocos ao acaso, com parcelas subdivididas e seis repetições. Os tratamentos consistiram de dois níveis de $\mathrm{CO}_{2}$ atmosférico, 390 e $550 \mu \mathrm{mol} \mathrm{mol}^{-1}$. A amostragem de solo foi realizada na linha e na entrelinha do cafeeiro, em 2013 e 2014, nas camadas de 0-5,0, 5,0-10, 10-20 e 20-40 cm, e de 0-10, 10-20 e 20-40 cm, respectivamente. Avaliaram-se $\mathrm{pH}$, teores de $\mathrm{Ca}^{2+}, \mathrm{Mg}^{2+}, \mathrm{K}, \mathrm{P}$ e S disponíveis, saturação por bases e matéria orgânica do solo. Em 2013 e 2014, houve redução nos teores de P na linha do cafeeiro, com o aumento da concentração de $\mathrm{CO}_{2}$. Em 2014, houve redução nos teores de $\mathrm{K}$ disponível no solo e aumento dos teores de $\mathrm{K}$ na folha do cafeeiro sob $550 \mu \mathrm{mol} \mathrm{mol}{ }^{-1} \mathrm{de} \mathrm{CO}_{2}$. Em cafeeiro cultivado em atmosfera enriquecida com $\mathrm{CO}_{2}$, o teor de $\mathrm{P}$ disponível no solo é o que mais reduz, o que indica a necessidade de reposição adequada deste nutriente.

Termos para indexação: Coffea arabica, carbono no solo, diagnose foliar, FACE, gases de efeito estufa, nutrientes.

\section{Soil fertility and nutritional status of coffee grown in $\mathrm{CO}_{2}$-enriched atmosphere}

\begin{abstract}
The objective of this work was to evaluate the effects of atmospheric $\mathrm{CO}_{2}$ levels on soil chemical attributes, in rows (coffee crop) and between rows (Urochloa decumbens), and on macronutrient contents in coffee leaves. A randomized complete block design was used, in a split-plot, with six replicates. The treatments consisted of two $\mathrm{CO}_{2}$ levels in the atmosphere, 390 and $550 \mu \mathrm{mol} \mathrm{mol}^{-1}$. Soil samples were collected in and between coffee rows in 2013 and 2014, at the soil layers of 0-5.0, 5.0-10, 10-20 and 20-40 cm, and of 0-10, 10-20 and 20-40 cm, respectively. Soil $\mathrm{pH}, \mathrm{Ca}^{2+}, \mathrm{Mg}^{2+}$, available $\mathrm{K}, \mathrm{P}$, and $\mathrm{S}$ contents, base saturation, and soil organic matter were evaluated. In 2013 and 2014, a reduction in available P contents was observed in coffee rows as the $\mathrm{CO}_{2}$ levels in the air were increased. In 2014, soil available $\mathrm{K}$ contents were reduced and $\mathrm{K}$ contents in coffee leaf were increased under $550 \mu \mathrm{mol} \mathrm{mol}^{-1} \mathrm{CO}_{2}$. In coffee grown in atmosphere enriched with $\mathrm{CO}_{2}$, soil available $\mathrm{P}$ content is more reduced, which indicates the need of an adequate replacement of this nutrient.

Index terms: Coffea arabica, soil carbon, leaf analysis, FACE, greenhouse gases, nutrients.
\end{abstract}

\section{Introdução}

O efeito estufa, causado pela presença de gases na atmosfera, como $\mathrm{CO}_{2}, \mathrm{CH}_{4}, \mathrm{~N}_{2} \mathrm{O}$, entre outros, é um fenômeno natural e essencial à vida no planeta. A partir da revolução industrial, a concentração de $\mathrm{CO}_{2}$ na atmosfera tem aumentado, com previsão, para 2100, de $700 \mu \mathrm{mol} \mathrm{mol}^{-1} \mathrm{de} \mathrm{CO}_{2}$ atmosférico (Intergovernmental Panel on Climate Change, 2013). Diante desse cenário, cabe à pesquisa agrícola verificar as respostas das plantas e de outras matrizes essenciais para seu cultivo, às concentrações cada vez maiores de $\mathrm{CO}_{2}$ no ar.
O café é uma das principais "commodities" agrícolas, já que sua cadeia de produção movimenta cerca de 90 bilhões de dólares em todo o mundo, com destaque para o Brasil, o maior produtor mundial (Amaral et al., 2011). Isso mostra a importância de estudos que avaliem as respostas do cafeeiro e de seus sistemas de cultivo ao acréscimo de $\mathrm{CO}_{2}$ na atmosfera. Ghini et al. (2015) verificaram aumento de 7,4\% em altura e de $9,5 \%$ em diâmetro do caule em cafeeiro 'Catuaí', sob concentração de $550 \mu \mathrm{mol} \mathrm{mol}{ }^{-1}$ de $\mathrm{CO}_{2}$, quando comparado às plantas cultivadas na concentração atual de $\mathrm{CO}_{2}$ no ar, de $390 \mu \mathrm{mol} \mathrm{mol}{ }^{-1}$. Como o $\mathrm{CO}_{2}$ 
é substrato primário para a fotossíntese, o aumento de sua concentração na atmosfera pode promover efeitos benéficos sobre as culturas, ao estimular a fotossíntese (Walter et al., 2015) e aumentar o crescimento e a produtividade (Sujatha et al., 2008). Contudo, há relatos de que plantas $\mathrm{C}_{3}$, como o cafeeiro, cultivadas em atmosfera enriquecida com $\mathrm{CO}_{2}$, apresentam maior crescimento e desenvolvimento do que plantas $\mathrm{C}_{4}$ (Poorter \& Navas, 2003), em razão do aumento da atividade fotossintética e da eficiência de uso da água (Ramalho et al., 2013).

Amaior produção de biomassa e de produtos colhidos de culturas submetidas à atmosfera enriquecida com $\mathrm{CO}_{2}$ não pode ser sustentada em solos deficientes ou com níveis atuais de nutrientes (Campbell \& Sage, 2006). Isso significa que, no futuro, poderá ocorrer aumento na aplicação de nutrientes, via adubação, para que haja incremento considerável no crescimento e na produtividade das culturas (Manoj-Kumar et al., 2011). Desse modo, é provável que esse novo padrão de crescimento vegetal, com elevação da concentração de $\mathrm{CO}_{2}$ atmosférico, aumente a exigência nutricional e a exportação de nutrientes pelas culturas, o que pode alterar o aporte de $\mathrm{C}$ e de outros nutrientes ao solo, com consequências diretas sobre a fertilidade do solo e exaustão mais rápida das reservas de nutrientes.

Em estudo realizado no sistema "free-air carbon dioxide enrichment" (FACE), plantas de trigo sob maior concentração de $\mathrm{CO}_{2}$ apresentaram aumento na produção, mas menor teor de proteínas nos grãos, uma vez que o trigo utilizou menos $\mathrm{N}$ na síntese de proteínas e mais na fotossíntese (Norton et al., 2011). Com maior oferta de $\mathrm{C}$, as plantas tendem a aumentar a eficiência de utilização do $\mathrm{N}$, pela maior produção e atividade da enzima ribulose 1,5-bifosfato carboxilase/ oxidase (Rubisco), o que resulta em manutenção ou redução nos teores de $\mathrm{N}$ foliar (Leakey et al., 2009), como descrito por Martins et al. (2014) em folhas de cafeeiro cultivado em $700 \mu \mathrm{mol} \mathrm{mol}{ }^{-1}$ de $\mathrm{CO}_{2}$. Esse efeito também ocorre em relação ao P (Manoj-Kumar et al., 2012), pois a Rubisco apresenta dois átomos de $P$ em sua composição e há maior demanda da planta por trifosfato de adenosina (ATP).

A maior produção de biomassa (parte aérea e radicular) das plantas em ambiente enriquecido com $\mathrm{CO}_{2}$ tende a aumentar os teores de $\mathrm{C}$ orgânico do solo (Hungate et al., 2009), o que contribui para o aumento da matéria orgânica e, consequentemente, pode aumentar a capacidade de troca de cátions. No Brasil, a dinâmica da matéria orgânica e dos nutrientes em solo cultivado com cafeeiro sob elevada concentração de $\mathrm{CO}_{2}$ é pouco entendida, o que requer o desenvolvimento de estudos para aperfeiçoar o manejo da fertilidade do solo e a nutrição mineral do cafeeiro.

O objetivo deste trabalho foi avaliar os efeitos de concentrações de $\mathrm{CO}_{2}$ atmosférico nos atributos químicos do solo, na linha (cafeeiro) e na entrelinha (braquiária), e nos teores de macronutrientes em folhas do cafeeiro.

\section{Material e Métodos}

O experimento foi conduzido na Embrapa Meio Ambiente, em Jaguariúna, SP $\left(22^{\circ} 43^{\prime} \mathrm{S}, 47^{\circ} 01^{\prime} \mathrm{W}\right.$, a $570 \mathrm{~m}$ de altitude), com cafeeiro cultivado no sistema FACE, internacionalmente conhecido para avaliações dos efeitos causados pelo aumento da concentração de $\mathrm{CO}_{2}$ em plantas cultivadas em campo. No presente trabalho, o experimento em FACE, denominado ClimapestFACE, é o primeiro do mundo realizado com cafeeiro (Ghini et al., 2011). A estrutura experimental e o cafeeiro foram implantados em 2011, em Latossolo Vermelho de textura argilosa. A temperatura média anual da região é de $21,7^{\circ} \mathrm{C}$ e a precipitação, de $1.335 \mathrm{~mm}$ por ano.

A área experimental ClimapestFACE ocupa 7,0 ha cultivados com café (Coffea arabica L.) 'Catuaí Vermelho IAC 144', em espaçamento de 3,60x0,60 m, com adubação anual na projeção da copa. Na entrelinha do cafeeiro, foi plantada braquiária [Urochloa decumbens (syn. Brachiaria decumbens)], sem adubação. A calagem e a adubação do cafeeiro, tanto na implantação quanto na manutenção anual, foram realizadas tendo como base a produtividade esperada de até 30 sacas por hectare, segundo recomendações de Raij et al. (1997).

O ClimapestFACE constitui-se de 12 parcelas, em formato octagonal, de $10 \mathrm{~m}$ de diâmetro cada uma, conduzidas em ambiente aberto. Os tratamentos foram: controle, seis parcelas mantidas na condição atual de concentração de $\mathrm{CO}_{2}$ na atmosfera, de $390 \pm 10 \mu \mathrm{mol} \mathrm{mol}{ }^{-1}$; e seis parcelas enriquecidas com $550 \pm 60 \mu \mathrm{mol} \mathrm{mol}{ }^{-1}$ de $\mathrm{CO}_{2}$. Cada parcela octogonal, com enriquecimento de $\mathrm{CO}_{2}$, apresenta válvulas em toda a extensão do octógono por onde o $\mathrm{CO}_{2}$ é injetado puro, com difusão natural do gás no ar. Essas válvulas de injeção de $\mathrm{CO}_{2}$ apresentam sistema automático de compensação da direção e da velocidade do 
vento, para promover distribuição uniforme do gás na parcela. A manutenção da concentração de $\mathrm{CO}_{2}$

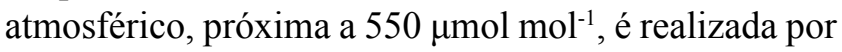
monitoramento em tempo real, por análise automática da concentração de $\mathrm{CO}_{2}$ pelo analisador de gás que opera na faixa do infravermelho (Irga), instalado no centro de cada parcela, ou seja, quando a concentração do gás no ar é reduzida, automaticamente há injeção de $\mathrm{CO}_{2}$ até $550 \mu \mathrm{mol} \mathrm{mol}{ }^{-1}$. Cada parcela tem bateria associada a um painel fotovoltaico, para fornecer energia aos equipamentos em operação no octógono.

Utilizou-se o delineamento experimental de blocos ao acaso com parcelas subdivididas no espaço e seis repetições. Os tratamentos consistiram de dois níveis de $\mathrm{CO}_{2}$ atmosférico, 390 e $550 \mu \mathrm{mol} \mathrm{mol}{ }^{-1}$, alocados nas parcelas, com dois locais de amostragem do solo, linha (cafeeiro) e entrelinha (braquiária), como subdivisão das parcelas. $\mathrm{O}$ solo foi coletado na linha e na entrelinha, em razão do cultivo simultâneo do cafeeiro na linha e da braquiária na entrelinha no ClimapestFACE, já que é provável que a magnitude de alterações nos atributos de fertilidade do solo seja diferente para cada local amostrado.

A amostragem de solo foi realizada em janeiro de 2013, nas camadas de $0-5,0,5,0-10,10-20$ e 20$40 \mathrm{~cm}$, e, em abril de 2014, nas camadas de $0-10,10$ 20 e $20-40 \mathrm{~cm}$, tanto na linha quanto na entrelinha do cafeeiro. Em cada subparcela (linha e entrelinha), foram coletadas cinco amostras simples do solo que, após misturadas, separadamente para cada profundidade, constituíram as amostras compostas utilizadas no laboratório para as determinações analíticas.

As amostras compostas de solo, coletadas em 2013 e 2014, foram secas em temperatura ambiente e passadas em peneira com malha de 2,0 $\mathrm{mm}$ de abertura, para avaliação dos atributos químicos do solo: $\mathrm{pH}$ em água 1:2,5 (v/v); teor de $\mathrm{H}+\mathrm{Al}$; teores de $\mathrm{P}$ (solução de Mehlich-1), $\mathrm{S}$ e $\mathrm{K}$ disponíveis, e de $\mathrm{Ca}^{2+}, \mathrm{Mg}^{2+}$ e $\mathrm{Al}^{3+}$, utilizados para cálculo da saturação por bases (V); e teores de matéria orgânica (MO) do solo. Nas amostras coletadas em 2014, foram analisados o teor de N-total, $\mathrm{N}-\mathrm{NH}_{4}{ }^{+}$e N-NO${ }_{3}^{-}$com o método Kjeldahl (Tedesco et al., 1995), além do teor de P disponível, extraído por resina de troca iônica mista (Raij et al., 2001).

Em janeiro de 2014, foram coletadas, em cada parcela, amostras de folha do cafeeiro do terceiro ou quarto par de folhas do ramo localizado no terço médio da planta. Essas amostras foram secas em estufa com circulação forçada de ar, a $60^{\circ} \mathrm{C}$, por 72 horas, e trituradas em moinho do tipo Willey com malha de 1,0 mm; em seguida, determinaram-se os teores de $\mathrm{N}$, após digestão sulfúrica, e de $\mathrm{P}, \mathrm{K}$ e $\mathrm{Mg}$, após digestão nitroperclórica na proporção de 4:1 (ácido nítrico:ácido perclórico).

Os dados foram submetidos à análise de variância, e as médias foram comparadas em função das concentrações de $\mathrm{CO}_{2}$ atmosférico e dos locais de amostragem do solo, pelo teste de Tukey, a 5\% de probabilidade, com uso do programa Sisvar, versão 5.3 (Ferreira, 2011).

\section{Resultados e Discussão}

As principais alterações nos atributos químicos foram observadas nas primeiras camadas de solo, tanto nas avaliações realizadas em 2013 quanto em 2014. Portanto, os resultados são apresentados e discutidos para as camadas amostradas entre 0 e $20 \mathrm{~cm}$ de perfil do solo, quanto aos atributos químicos que apresentaram diferenças significativas, em função das concentrações de $\mathrm{CO}_{2}$, do local de amostragem do solo ou da interação entre estes dois fatores.

$\mathrm{O} \mathrm{pH}$ e os teores de $\mathrm{S}$, na camada de 0 a $5,0 \mathrm{~cm}$, e de $\mathrm{P}$ e $\mathrm{S}$, na de 5,0 a $10 \mathrm{~cm}$, avaliados em 2013, foram influenciados apenas pela concentração de $\mathrm{CO}_{2}$ atmosférico (Tabela 1). Os maiores valores de $\mathrm{pH}$ foram encontrados no solo com plantas cultivadas sob concentração de $550 \mu \mathrm{mol} \mathrm{mol}^{-1}$ de $\mathrm{CO}_{2}$ do que sob a condição atual de $\mathrm{CO}_{2}$ atmosférico, de $390 \mu \mathrm{mol} \mathrm{mol}^{-1}$. $\mathrm{O}$ maior crescimento do cafeeiro, em resposta ao enriquecimento do ar com $\mathrm{CO}_{2}$, foi constatado por Ghini et al. (2015) em fase anterior do experimento ClimapestFACE. O enriquecimento com $\mathrm{CO}_{2}$ pode

Tabela 1. Valores de $\mathrm{pH}$ e teores de $\mathrm{S}$ disponível, na camada de $0-5,0 \mathrm{~cm}$ do solo, e teores de $\mathrm{Pe} \mathrm{S}$ disponíveis, na camada de 5,0-10 cm, em 2013, em função da concentração de $\mathrm{CO}_{2}$ na atmosfera ${ }^{(1)}$.

\begin{tabular}{lcc}
\hline Atributo & \multicolumn{2}{c}{ Concentração de $\mathrm{CO}_{2}$ na atmosfera $\left(\mu \mathrm{mol} \mathrm{mol}^{-1}\right)$} \\
\cline { 2 - 3 } & 390 & 550 \\
\hline & Camada de $0-5,0 \mathrm{~cm}$ do solo \\
pH em água & $5,5 \mathrm{~b}$ & $5,9 \mathrm{a}$ \\
$\mathrm{S}$ disponível $\left(\mathrm{mg} \mathrm{dm}^{-3}\right)$ & $12,2 \mathrm{a}$ & $8,0 \mathrm{~b}$ \\
\hline & Camada de $5,0-10 \mathrm{~cm}$ do solo \\
P Mehlich-1 $\left(\mathrm{mg} \mathrm{dm}^{-3}\right)$ & $2,1 \mathrm{a}$ & $1,6 \mathrm{~b}$ \\
S disponível $\left(\mathrm{mg} \mathrm{dm}^{-3}\right)$ & $8,0 \mathrm{a}$ & $6,5 \mathrm{~b}$ \\
\hline
\end{tabular}

${ }^{(1)}$ Médias seguidas de letras iguais não diferem pelo teste de Tukey, a 5\% de probabilidade.

Pesq. agropec. bras., Brasília, v.50, n.11, p.1087-1096, nov. 2015 DOI: 10.1590/S0100-204X2015001100012 
ter acelerado o processo de ciclagem interna de nutrientes na planta, a renovação dos tecidos vegetais e a deposição de resíduos, principalmente folhas sobre a superfície do solo, cuja decomposição pode aumentar o pH nas primeiras camadas de solo. A tendência da decomposição de resíduos vegetais é reduzir a acidez do solo; a acidificação normalmente ocorre durante a decomposição de resíduos vegetais com maiores teores de $\mathrm{N}$, uma vez que o processo de nitrificação promove liberação de $\mathrm{H}^{+}$e redução do $\mathrm{pH}$ do solo (Yan et al., 1996). Além disso, o aumento do $\mathrm{pH}$ também pode ser ocasionado pela complexação de $\mathrm{H}^{+} \mathrm{e}^{3 l^{3+}}$ por ácidos orgânicos liberados durante a decomposição de resíduos vegetais e da MO do solo (Pavinato \& Rosolem, 2008).

Em relação ao $\mathrm{S}$ e $\mathrm{P}$ disponíveis, menores teores no solo foram observados na condição de maior concentração de $\mathrm{CO}_{2}$, em comparação à condição atual do gás no ar (Tabela 1), o que pode estar relacionado ao aumento na absorção desses nutrientes pelas plantas de cafeeiro e braquiária, em resposta ao enriquecimento do ar com $\mathrm{CO}_{2}$. Ghini et al. (2015) observaram que plantas de café cultivadas no ClimapestFACE aumentaram a taxa de assimilação de $\mathrm{CO}_{2}$ em atmosfera enriquecida com o gás, em comparação às sob concentração de $390 \mu \mathrm{mol} \mathrm{mol}^{-1}$ de $\mathrm{CO}_{2}$ atmosférico. Assim, quando se aumenta a quantidade de $\mathrm{C}$ na planta, é possível que haja acréscimo na absorção de nutrientes, com consequente redução na disponibilidade destes no solo (Campbell \& Sage, 2006).

O local de amostragem também influenciou alguns atributos químicos do solo, independentemente da concentração de $\mathrm{CO}_{2}$, nas diferentes camadas amostradas, principalmente em 2014 (Tabela 2), o que mostra que essas mudanças estão relacionadas às plantas cultivadas, isto é, cafeeiro na linha e braquiária na entrelinha.

Em 2014, os teores de $\mathrm{Ca}^{2+}$, na camada de 0 a $10 \mathrm{~cm}$, e de $\mathrm{Mg}^{2+}, \mathrm{pH}$ e saturação por bases, tanto na camada de 0 a 10 quanto na de 10 a $20 \mathrm{~cm}$ do solo, apresentaram menores valores na linha de plantio (Tabela 2).

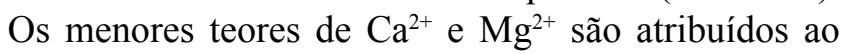
aumento na absorção destes nutrientes pelo cafeeiro, explicado pelo início da fase de produção do cafeeiro em 2014, o terceiro ano da sua implantação. Valarini et al. (2005), ao revisar trabalhos relacionados à nutrição do cafeeiro, constataram que, de 2 para 3 anos de idade, o cafeeiro duplica a exigência em nutrientes, especialmente em $\mathrm{N}, \mathrm{K}$ e $\mathrm{Ca}$, em razão do início da produção de grãos. Essas reduções nos teores de $\mathrm{Ca}^{2+}$ e $\mathrm{Mg}^{2+}$ favoreceram a diminuição dos valores de $\mathrm{pH}$ do solo na linha, o que promoveu maior atividade do $\mathrm{H}^{+}$na solução do solo. Nas camadas de solo menos estratificadas, a acidificação causada pela maior absorção de cátions no solo pode ser dominante, o que sobrepõe o efeito corretivo da acidez que pode resultar da decomposição dos resíduos vegetais.

Menores teores de MO no solo da linha do cafeeiro foram encontrados nas camadas de 0 a 10 e 10 a $20 \mathrm{~cm}$, em 2014 (Tabela 2), provavelmente em decorrência da adubação, que, além de fornecer nutrientes ao cafeeiro, estimula os microrganismos a decompor o $\mathrm{C}$ do solo. $\mathrm{O}$ inverso foi observado para $\mathrm{S}$ disponível, uma vez que a decomposição da $\mathrm{MO}$ do solo fornece $\mathrm{S}$ para o meio. Teores de $\mathrm{S}$ disponível nos solos, geralmente, estão relacionados à $\mathrm{MO}$, dado que a mineralização da MO do solo contribui para o aumento do teor de S disponível (Carmona et al., 2009).

Foram verificados efeitos da interação concentração de $\mathrm{CO}_{2}$ e local de amostragem do solo, principalmente nas coletas realizadas em 2013 (Figura 1). Neste ano, os teores de $\mathrm{K}$ disponível, na camada de 0 a $5 \mathrm{~cm}$ do solo, reduziram na entrelinha, com o aumento da concentração de $\mathrm{CO}_{2}$. Além disso, quando comparadas ao cafeeiro, cujo crescimento inicial é lento, plantas $\mathrm{C}_{4}$,

Tabela 2. Valores de $\mathrm{pH}$ e teores de $\mathrm{Ca}^{2+}, \mathrm{Mg}^{2+}, \mathrm{S}$ disponível, matéria orgânica (MO) e saturação por bases $(\mathrm{V})$, na camada de 0-10 cm do solo, bem como $\mathrm{pH}$, teores de $\mathrm{Mg}^{2+}, \mathrm{K}$ e S disponíveis, MO e V, na camada de 10-20 cm, em 2014, em função do local de amostragem do solo ${ }^{(1)}$.

\begin{tabular}{|c|c|c|}
\hline \multirow[t]{2}{*}{ Atributo } & \multicolumn{2}{|c|}{ Local de amostragem do solo ${ }^{(2)}$} \\
\hline & Linha & Entrelinha \\
\hline & \multicolumn{2}{|c|}{ Camada de $0-10 \mathrm{~cm}$ do solo } \\
\hline $\mathrm{pH}$ em água & $4,8 b$ & $5,5 \mathrm{a}$ \\
\hline $\mathrm{Ca}^{2+}\left(\mathrm{cmol}_{\mathrm{c}} \mathrm{dm}^{-3}\right)$ & $1,4 \mathrm{~b}$ & $2,9 \mathrm{a}$ \\
\hline $\mathrm{Mg}^{2+}\left(\mathrm{cmol}_{\mathrm{c}} \mathrm{dm}^{-3}\right)$ & $0,8 \mathrm{~b}$ & $2,4 \mathrm{a}$ \\
\hline $\mathrm{S}$ disponível $\left(\mathrm{mg} \mathrm{dm}^{-3}\right)$ & $10,9 \mathrm{a}$ & $6,5 b$ \\
\hline $\mathrm{MO}\left(\mathrm{g} \mathrm{kg}^{-1}\right)$ & $2,2 b$ & $2,8 \mathrm{a}$ \\
\hline \multirow[t]{2}{*}{ V $(\%)$} & $34,4 \mathrm{~b}$ & $58,9 \mathrm{a}$ \\
\hline & \multicolumn{2}{|c|}{ Camada de $10-20 \mathrm{~cm}$ do solo } \\
\hline pH em água & $4,9 b$ & $5,7 \mathrm{a}$ \\
\hline $\mathrm{Mg}^{2+}\left(\mathrm{cmol}_{\mathrm{c}} \mathrm{dm}^{-3}\right)$ & $1,0 \mathrm{~b}$ & $2,2 \mathrm{a}$ \\
\hline $\mathrm{K}$ disponível (mg dm²) & $160,7 \mathrm{a}$ & $129,8 b$ \\
\hline $\mathrm{S}$ disponível (mg dm-3) & $11,3 \mathrm{a}$ & $6,8 \mathrm{~b}$ \\
\hline MO (\%) & $2,0 \mathrm{~b}$ & $2,4 a$ \\
\hline V (\%) & $40,5 b$ & $62,1 \mathrm{a}$ \\
\hline
\end{tabular}

${ }^{(1)}$ Médias seguidas de letras iguais não diferem pelo teste de Tukey, a 5\% de probabilidade. ${ }^{(2)}$ Linha, plantio de café (Coffea arabica); e entrelinha, plantio de braquiária (Urochloa decumbens). 
Camada de $0-5,0 \mathrm{~cm}$ do solo, ano 2013
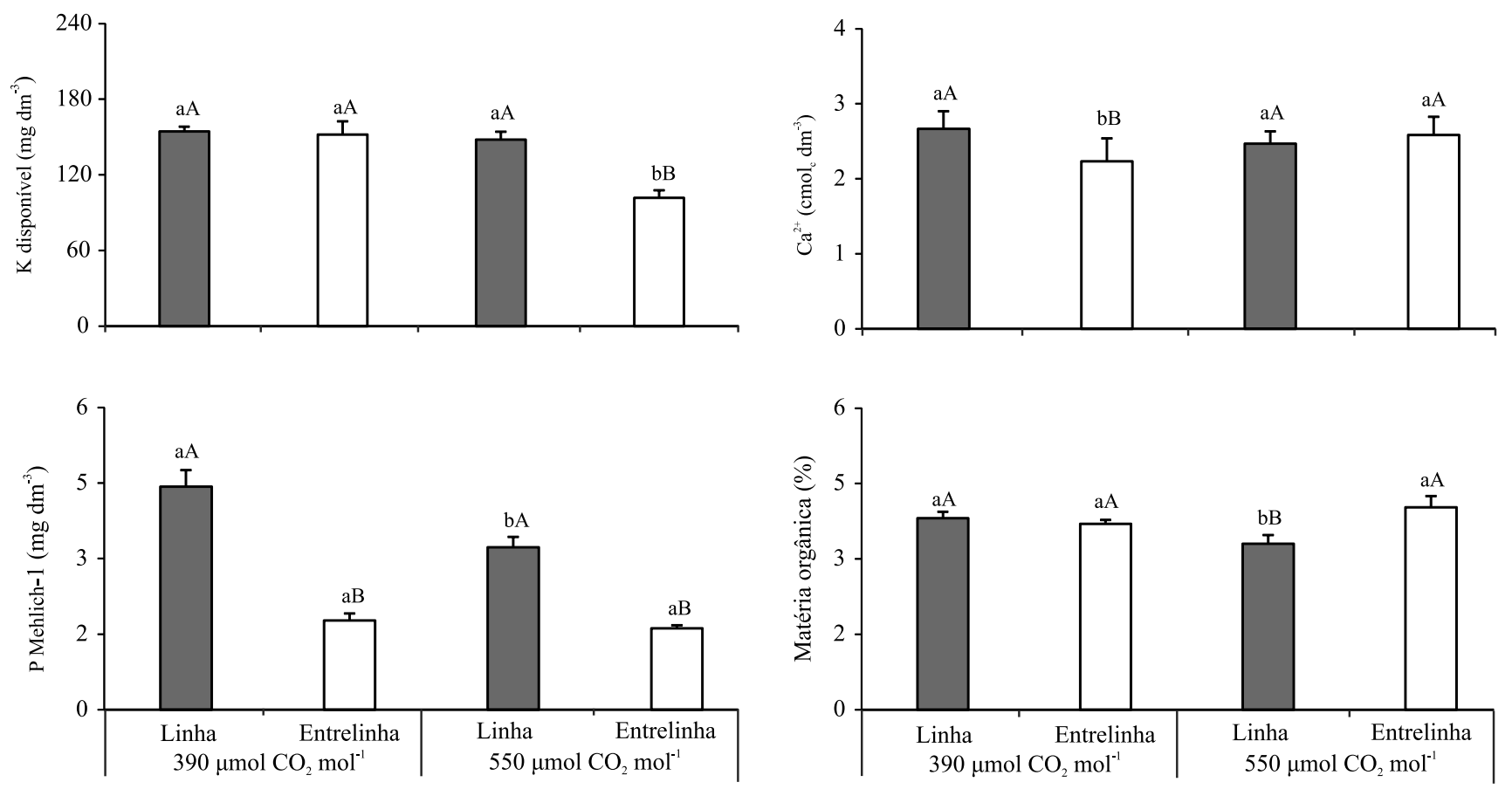

Camada de 5,0-10,0 cm do solo, ano 2013
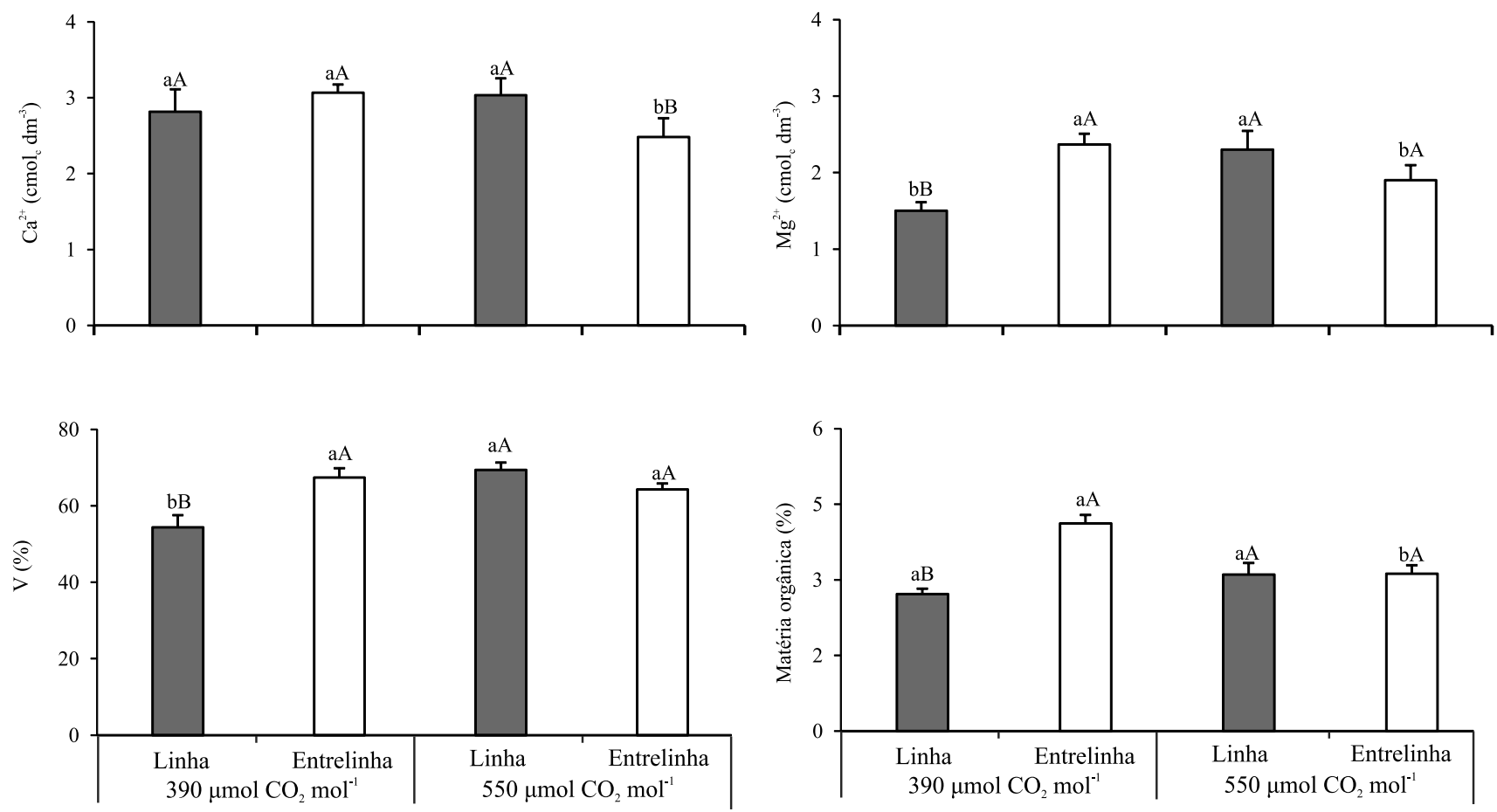

Figura 1. Teores de K disponível, $\mathrm{Ca}^{2+}$, P-Mehlich-1 e matéria orgânica (MO), na camada de 0-5,0 cm do solo; teores de $\mathrm{Ca}^{2+}, \mathrm{Mg}^{2+}$, saturação por bases (V) e $\mathrm{MO}$, na camada de 5,0-10,0 cm, em 2013, em função da concentração de $\mathrm{CO}_{2}$ e do local de amostragem do solo. Barras seguidas de letras iguais, minúsculas (concentrações de $\mathrm{CO}_{2}$ ) e maiúsculas (local de amostragem do solo), não diferem pelo teste de Tukey, a 5\% de probabilidade. 
como a braquiária, também podem apresentar maior crescimento na condição de ar enriquecido com $\mathrm{CO}_{2}$ (Poorter\&Navas, 2003), o quepode aumentaraabsorção de $\mathrm{K}^{+}$do solo. Plantas do gênero Urochloa apresentam grande capacidade de absorção e acumulação de $\mathrm{K}$ em seus tecidos, superior a de N (Rosolem et al., 2012). Entretanto, em 2014, na camada de 0 a $10 \mathrm{~cm}$ do solo, foram observados menores teores de $\mathrm{K}$ disponível na linha do cafeeiro com $550 \mu \mathrm{mol} \mathrm{mol}{ }^{-1}$ de $\mathrm{CO}_{2}$, em comparação à concentração de $390 \mu \mathrm{mol} \mathrm{mol}^{-1}$; esse efeito, inverso ao constatado em 2013, pode estar relacionado ao aumento da absorção de $\mathrm{K}$ pelo cafeeiro, pois, na fase de início da produção de grãos (terceiro ano de implantação do cafeeiro no ClimapestFACE), a exigência em $\mathrm{K}$ também é duplicada e pode ser maior em condição de elevada concentração de $\mathrm{CO}_{2}$. Nas camadas de 5,0-10 e 10-20, em 2013, foram observadas reduções nos teores de $\mathrm{Ca}^{2+}$ e de $\mathrm{Mg}^{2+}$, na entrelinha do cafeeiro cultivado com $550 \mu \mathrm{mol} \mathrm{mol}^{-1}$ de $\mathrm{CO}_{2}$ (Figuras 1 e 2). Esse efeito é indicação de que, em condição de elevada concentração de $\mathrm{CO}_{2}, \mathrm{o}$ crescimento e o desenvolvimento iniciais da braquiária podem ser maiores do que na condição atual de $\mathrm{CO}_{2}$ do ar, o que causa maior absorção desses nutrientes.

Os teores de $\mathrm{P}$ disponível na linha do cafeeiro, avaliados em 2013 e 2014, foram menores com $550 \mu \mathrm{mol} \mathrm{mol}{ }^{-1}$ do que com $390 \mu \mathrm{mol} \mathrm{mol}^{-1}$ de $\mathrm{CO}_{2}$ (Figuras 1, 2 e 3), o que reforça a influência do

Camada de 10-20,0 cm do solo, ano 2013
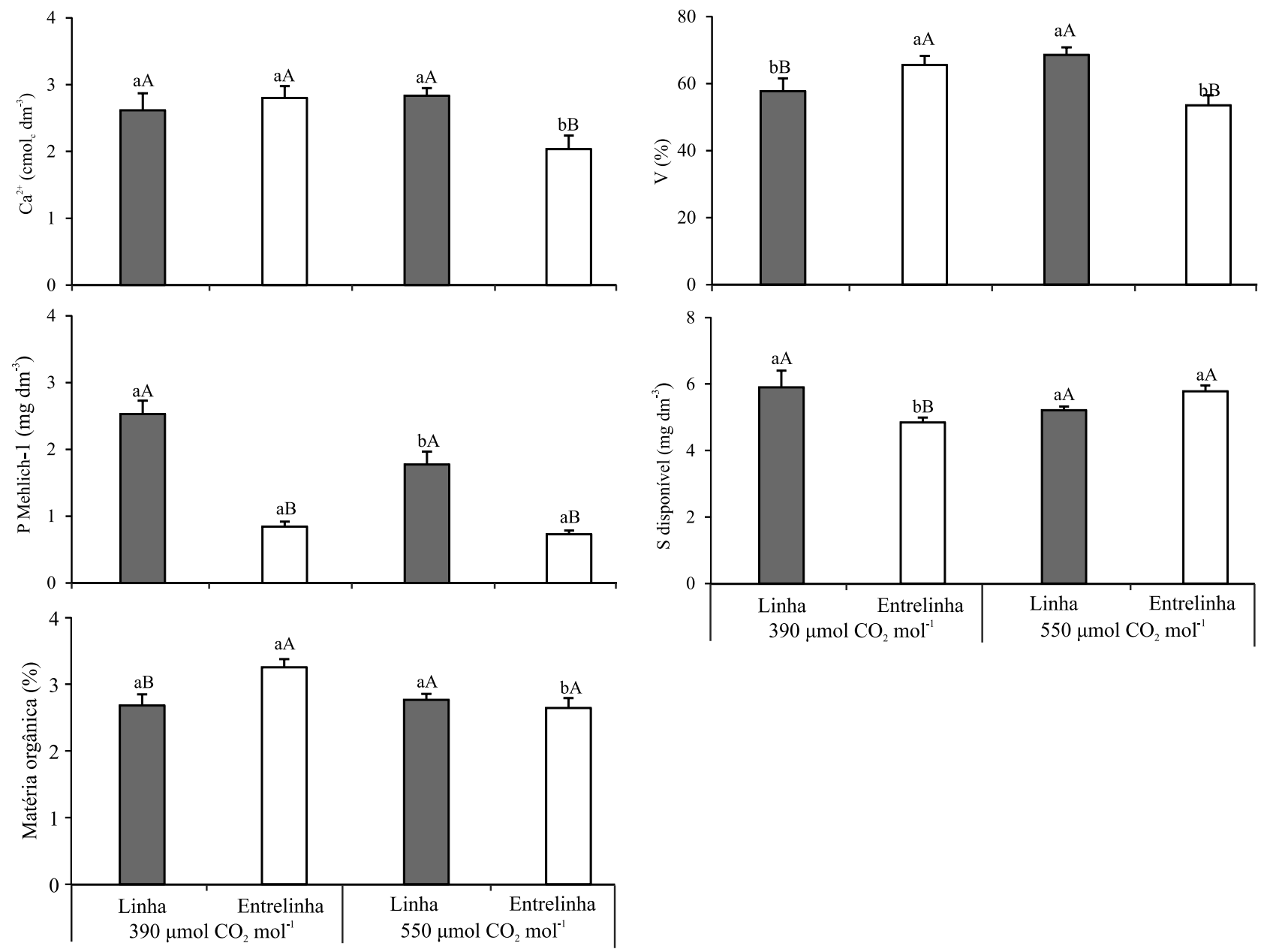

Figura 2. Teores de $\mathrm{Ca}^{2+}$, saturação por bases (V), P Mehlich-1, S disponível e matéria orgânica, na camada de 10-20 cm, em 2013, em função da concentração de $\mathrm{CO}_{2}$ e do local de amostragem do solo. Barras seguidas de letras iguais, minúsculas (concentrações de $\mathrm{CO}_{2}$ ) e maiúsculas (local de amostragem do solo), não diferem pelo teste de Tukey, a 5\% de probabilidade. 

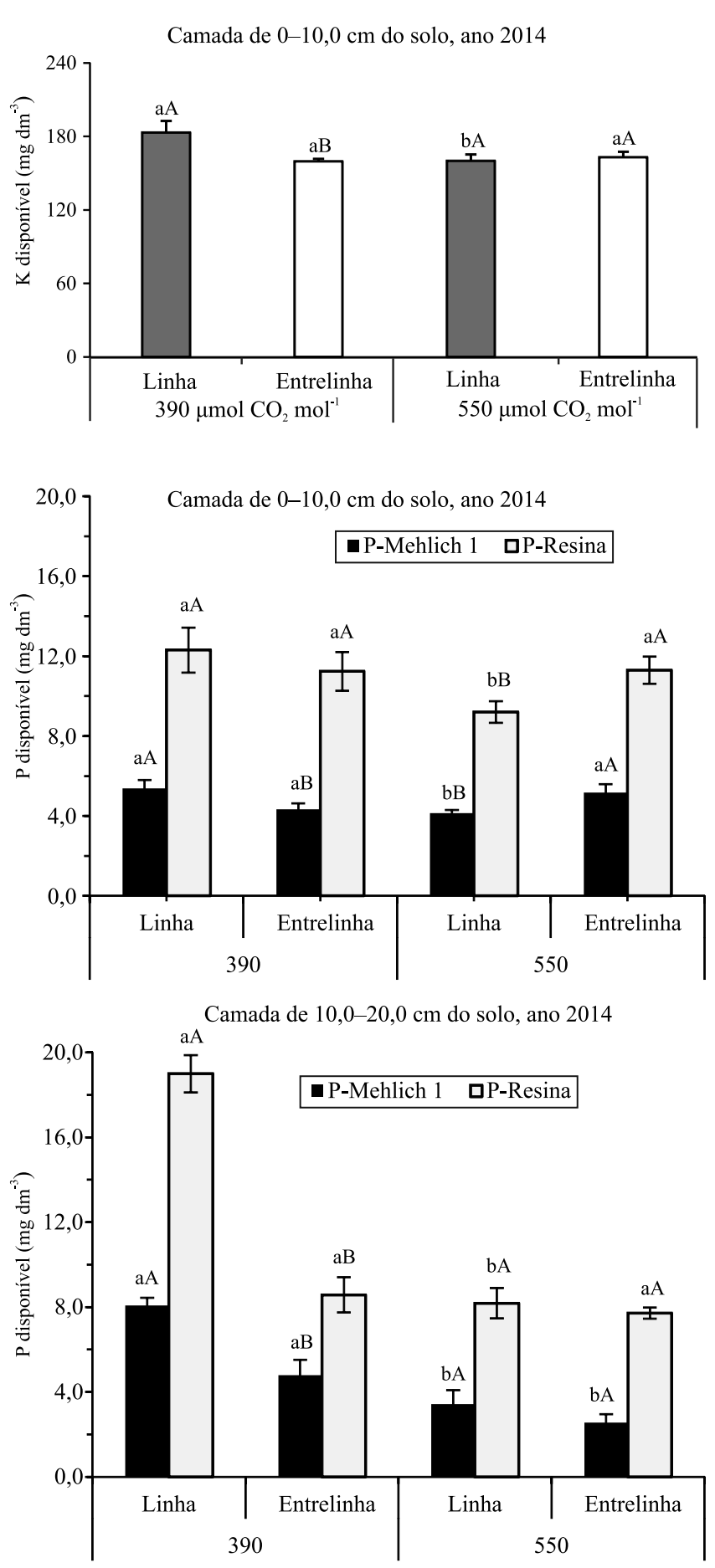

Figura 3. Teores de K e P disponíveis, extraídos com solução de Mehlich-1 e resina, na camada de $0-10 \mathrm{~cm}$ do solo; e teores de P disponível extraído com Mehlich-1 e resina, na camada de 10-20 cm, em 2014, em função da concentração de $\mathrm{CO}_{2}$ e do local de amostragem do solo. Barras seguidas de letras iguais, minúsculas (concentrações de $\mathrm{CO}_{2}$ ) e maiúsculas (local de amostragem do solo), não diferem pelo teste de Tukey, a $5 \%$ de probabilidade. aumento da concentração de $\mathrm{CO}_{2}$ sobre a absorção de P pelo cafeeiro. Langley \& Megonigal (2010) observaram aumento no crescimento de plantas $\mathrm{C}_{3}$ sob enriquecimento da atmosfera com $\mathrm{CO}_{2}$, embora esse efeito tenha sido maior quando a dose de $\mathrm{N}$ foi elevada, o que evidencia aumento na exigência de $\mathrm{N}$ pelas plantas. Assim como o N, o P pode exercer importante controle sobre o comportamento de plantas cultivadas em ambiente com enriquecimento do ar com $\mathrm{CO}_{2}$. Manoj-Kumar et al. (2012) relataram aumento de 59\% na absorção de $\mathrm{P}$ e de $74 \%$ na taxa de recuperação do $\mathrm{P}$ aplicado, via fertilizante, por plantas de trigo (Triticum aestivum L.) cultivadas em ar com $650 \mu \mathrm{mol} \mathrm{mol}^{-1} \mathrm{de}$ $\mathrm{CO}_{2}$, quando comparado à condição atual do gás na atmosfera.

Em experimentos FACE com diversas culturas sob concentração de $\mathrm{CO}_{2}$ elevada, tem-se observado crescimento do sistema radicular superior ao da parte aérea, principalmente onde há cultivo de plantas $\mathrm{C}_{3}$ (Kimball et al., 2002). Isso resulta na exploração de maior volume de solo pelas plantas e no favorecimento da absorção de nutrientes, como K e P, transportados no solo predominantemente por difusão.

$\mathrm{O}$ teor de $\mathrm{MO}$ foi menor na linha do café, com o aumento da concentração de $\mathrm{CO}_{2}$ na camada de 0-5,0 cm, em 2013 (Figura 1), possivelmente pelo aumento do aporte de resíduos vegetais à superfície do solo, o que estimula a atividade dos microrganismos e acelera a decomposição da MO. Fontaine et al. (2007) relataram aumento na proporção de C lábil em resíduos vegetais com a elevação da concentração de $\mathrm{CO}_{2}$, o que estimula ainda mais a decomposição da MO presente no solo. Nesse mesmo ano, as mudanças nos teores de $\mathrm{S}$ disponível no solo, na camada de 10-20 cm, apresentaram relação inversa com as alterações observadas na MO. Na entrelinha com $550 \mu \mathrm{mol} \mathrm{mol}{ }^{-1}$ de $\mathrm{CO}_{2}$, houve redução no teor de $\mathrm{MO}$, o que pode ter proporcionado aumento no teor de S disponível.

Nas análises de macronutrientes do tecido foliar do cafeeiro, foram encontradas diferenças apenas nos teores de $\mathrm{K}$ e $\mathrm{Mg}$, em função da concentração de $\mathrm{CO}_{2}$ (Tabela 3); no entanto, em ambas as concentrações de $\mathrm{CO}_{2}$ no ar, os teores desses nutrientes foram considerados adequados para o cafeeiro, de acordo com os valores preconizados por Malavolta et al. (1997). Na condição de $550 \mu \mathrm{mol} \mathrm{mol}^{-1}$ de $\mathrm{CO}_{2}$ no ar, o teor foliar de $\mathrm{K}$ do cafeeiro foi maior do que na de

Pesq. agropec. bras., Brasília, v.50, n.11, p.1087-1096, nov. 2015 DOI: 10.1590/S0100-204X2015001100012 
$390 \mu \mathrm{mol} \mathrm{mol}{ }^{-1}$; isso explica a redução no teor de $\mathrm{K}$ disponível da camada de $0-10 \mathrm{~cm}$ do solo, na coleta em 2014 (Figura 3), o que confirma a maior absorção desse nutriente pelo cafeeiro. Em relação ao $\mathrm{Mg}$, entretanto, houve redução no teor foliar na condição de $550 \mu \mathrm{mol} \mathrm{mol}{ }^{-1}$ de $\mathrm{CO}_{2}$, possivelmente pela maior eficiência de utilização de $\mathrm{Mg}$ pelo cafeeiro. Ramalho et al. (2013), em estudo com genótipos de cafeeiro e concentrações de $\mathrm{CO}_{2}$ no ar, verificaram maior taxa de assimilação de $\mathrm{CO}_{2}$ em cafeeiro cultivado em $700 \mu \mathrm{mol} \mathrm{mol}^{-1}$ de $\mathrm{CO}_{2}$ atmosférico, do que em $380 \mu \mathrm{mol} \mathrm{mol}{ }^{-1}$, o que representa aumento da capacidade fotossintética e da produção de fotoassimilados na planta. Assim, maior produção de clorofila, cujo Mg é um dos constituintes, pode ter ocorrido no cafeeiro, o que acarreta redistribuição e diluição do $\mathrm{Mg}$ nos tecidos da planta, bem como redução no teor do nutriente na folha, em ambiente enriquecido com $\mathrm{CO}_{2}$.

A concentração de $\mathrm{CO}_{2}$ no ar não produziu efeito sobre o teor de $\mathrm{N}$ foliar do cafeeiro (Tabela 3), nem sobre os teores de $\mathrm{N}$-total, $\mathrm{N}_{-} \mathrm{NH}_{4}{ }^{+}$e $\mathrm{N}^{-\mathrm{NO}_{3}}{ }^{-}$no solo, nas amostras coletadas em 2014. O N tem sido indicado como o nutriente que mais se relaciona ao crescimento, ao desenvolvimento e à produtividade das culturas em condições de cultivo com elevado teor de $\mathrm{CO}_{2}$ atmosférico (Walter et al., 2015); contudo, isso tem sido observado em estudos com culturas de ciclo de vida anual (Manoj-Kumar et al., 2011; Norton et al., 2011). Desse modo, é provável que, para plantas de ciclo perene com crescimento inicial lento, como o cafeeiro, essa relação ocorra em razão do aumento da produção de grãos e da exigência da planta por $\mathrm{N}$, especialmente em cultivares de maior porte ou com maior potencial produtivo, dependentemente do genótipo cultivado. Martins et al. (2014) constataram

Tabela 3. Teores de K, Mg, N e P em folhas de café (Coffea arabica), em função da concentração de $\mathrm{CO}_{2}{ }^{(1)}$.

\begin{tabular}{lcc}
\hline $\begin{array}{l}\text { Nutriente na folha } \\
\left(\mathrm{g} \mathrm{kg}^{-1}\right)\end{array}$ & \multicolumn{2}{c}{ Concentração de $\mathrm{CO}_{2}$ na atmosfera $\left(\mu \mathrm{mol} \mathrm{mol}{ }^{-1}\right)$} \\
\cline { 2 - 3 } $\mathrm{K}$ & 390 & 550 \\
$\mathrm{Mg}$ & $15,2 \mathrm{~b}$ & $16,1 \mathrm{a}$ \\
$\mathrm{N}$ & $4,6 \mathrm{a}$ & $4,0 \mathrm{~b}$ \\
$\mathrm{P}$ & $34,0 \mathrm{a}$ & $34,0 \mathrm{a}$ \\
\hline
\end{tabular}

${ }^{(1)}$ Médias seguidas de letras iguais não diferem pelo teste de Tukey, a $5 \%$ de probabilidade. que os teores de $\mathrm{N}$ foliar do cafeeiro diminuíram em dois dos três genótipos cultivados em $700 \mu \mathrm{mol} \mathrm{mol}^{-1}$ de $\mathrm{CO}_{2}$ no ar. Dessa forma, espera-se observar, na fase de plena produção do cafeeiro, efeito de demanda magnificada de $\mathrm{N}$.

$\mathrm{O}$ teor foliar de $\mathrm{P}$ do cafeeiro não foi influenciado pela concentração de $\mathrm{CO}_{2}$ (Tabela 3), embora avaliações feitas no solo apresentassem evidências de que houve maior absorção de $\mathrm{P}$ pelo cafeeiro cultivado em $550 \mu \mathrm{mol} \mathrm{mol}^{-1}$ do gás (Figuras 1, $2 \mathrm{e}$ 3). Manoj-Kumar et al. (2012) observaram aumento na absorção de $\mathrm{P}$ por plantas de trigo cultivadas em ar com

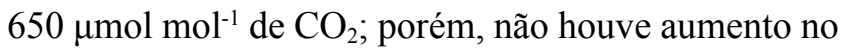
teor de $\mathrm{P}$ da raiz, da folha e do caule, apenas dos grãos. Assim, o P absorvido pode ter sido redistribuído na planta, para ser utilizado em compostos orgânicos de outras partes do vegetal, o que evitou o acúmulo de $\mathrm{P}$ na folha amostrada.

\section{Conclusões}

1. O aumento da concentração de $\mathrm{CO}_{2}$ reduz a disponibilidade de $\mathrm{K}$, em camadas superficiais, e de $\mathrm{P}$, em todas as camadas, até $20 \mathrm{~cm}$ de profundidade do solo, na linha do cafeeiro (Coffea arabica), o que indica a necessidade de reposição adequada deste nutriente.

2. Com o aumento da concentração de $\mathrm{CO}_{2}$ na

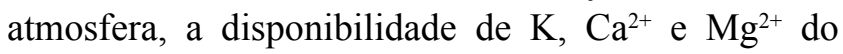
solo diminui na entrelinha do cafeeiro cultivado com braquiária (Urochloa decumbens).

3. Os teores de $\mathrm{K}$ aumentam e os de $\mathrm{Mg}$ diminuem em folhas de cafeeiro cultivado em atmosfera enriquecida com $\mathrm{CO}_{2}$.

\section{Agradecimentos}

Ao Conselho Nacional de Desenvolvimento Científico e Tecnológico (CNPq), à Coordenação de Aperfeiçoamento de Pessoal de Nível Superior (Capes) e à Fundação de Amparo à Pesquisa do Estado de Minas Gerais (Fapemig), pela concessão de bolsas; e aos pesquisadores José Ricardo Pupo Gonçalves (in memoriam), Gilmar Victorino, Kátia de Lima Nechet e à equipe da Embrapa Meio Ambiente, pela coordenação, pela montagem, pela condução e pela manutenção do experimento ClimapestFACE. 


\section{Referências}

AMARAL, J.F.T. do; MARTINEZ, H.E.P.; LAVIOLA, B.G.; FERNANDES FILHO, E.I.; CRUZ, C.D. Eficiência de utilização de nutrientes por cultivares de cafeeiro. Ciência Rural, v.41, p.621-629, 2011. DOI: 10.1590/S0103-84782011005000027.

CAMPBELL, C.D.; SAGE, R.E. Interactions between the effects of atmospheric $\mathrm{CO}_{2}$ content and $\mathrm{P}$ nutrition on photosynthesis in white lupin (Lupinus albus L.). Plant, Cell and Environment, v.29, p.844-853, 2006. DOI: 10.1111/j.1365-3040.2005.01464.x.

CARMONA, F. de C.; CONTE, O.; FRAGA, T.I.; BARROS, T.; PULVER, E.; ANGHINONI, I. Disponibilidade no solo, estado nutricional e recomendação de enxofre para o arroz irrigado. Revista Brasileira de Ciência do Solo, v.33, p.345-355, 2009. DOI: 10.1590/S0100-06832009000200012.

FERREIRA, D.F. Sisvar: a computer statistical analysis system. Ciência e Agrotecnologia, v.35, p.1039-1042, 2011.

FONTAINE, S.; BAROT, S.; BARRÉ, P.; BDIOUI, N.; MARY, B.; RUMPEL, C. Stability of organic carbon in deep soil layers controlled by fresh carbon supply. Nature, v.450, p.277-280, 2007. DOI: 10.1038 /nature06275.

GHINI, R.; BETTIOL, W.; HAMADA, E. Diseases in tropical and plantation crops as affected by climate changes: current knowledge and perspectives. Plant Pathology, v.60, p.122-132, 2011. DOI: 10.1111/j.1365-3059.2010.02403.x.

GHINI, R.; TORRE-NETO, A.; DENTZIEN, A.F.M.; GUERREIRO-FILHO, O.; IOST, R.; PATRÍCIO, F.R.A.; PRADO, J.S.M.; THOMAZIELLO, R.A.; BETTIOL, W.; DAMATTA, F.M. Coffee growth, pest and yield responses to free-air $\mathrm{CO}_{2}$ enrichment. Climatic Change, v.32, p.307-320, 2015. DOI: 10.1007/s10584-015-1422-2.

HUNGATE, B.A.; GROENIGEN, K.J. van; SIX, J.; JASTROW, J.D.; LUO, Y.; GRAAFF, M.A. de; KESSEL, C. van; OSENBERG, C.W. Assessing the effect of elevated carbon dioxide on soil carbon: a comparison of four meta-analyses. Global Change Biology, v.15, p.2020-2034, 2009. DOI: 10.1111/j.1365-2486.2009.01866.x.

INTERGOVERNMENTAL PANEL ON CLIMATE CHANGE. Climate change 2013: the physical science basis: working group 1 contribution to the fifth assessment report of the Intergovernmental Panel on Climate Change. New York: Cambridge University, 2013. $1535 \mathrm{p}$.

KIMBALL, B.A.; KOBAYASHI, K.; BINDI, M. Responses of agricultural crops to free-air $\mathrm{CO}_{2}$ enrichment. Advances in Agronomy, v.77, p.293-368, 2002. DOI: 10.1016/ S0065-2113(02)77017-X.

LANGLEY, J.A.; MEGONIGAL, J.P. Ecosystem response to elevated $\mathrm{CO}_{2}$ levels limited by nitrogen-induced plant species shift. Nature, v.466, p.96-99, 2010. DOI: 10.1038/nature09176.

LEAKEY, A.D.B.; AINSWORTH, E.A.; BERNACCHI, C.J.; ROGERS, A.; LONG, S.P.; ORT, D.R. Elevated $\mathrm{CO}_{2}$ effects on plant carbon, nitrogen and water relations: six important lessons from FACE. Journal of Experimental Botany, v.60, p.2859-2876, 2009. DOI: $10.1093 / \mathrm{jxb} / \mathrm{erp} 096$.
MALAVOLTA, E.; VITTI, G.C.; OLIVEIRA, S.A. de. Avaliação do estado nutricional das plantas: princípios e aplicações. 2.ed. Piracicaba: Potafos, 1997. 319p.

MANOJ-KUMAR; PATRA, A.K.; SWARUP, A. Impact of climate change on fertilizer demand in agriculture: concerns and imperatives for food security in India. Indian Journal of Fertilizers, v.7, p.48-62, 2011.

MANOJ-KUMAR; SWARUP, A.; PATRA, A.K.; CHANDRAKALA, J.U.; MANJAIAH, K.M. Effect of elevated $\mathrm{CO}_{2}$ and temperature on phosphorus efficiency of wheat grown in an Inceptisol of subtropical India. Plant, Soil and Environment, v.58, p.230-235, 2012.

MARTINS, L.D.; TOMAZ, M.A.; LIDON, F.C.; DAMATTA, F.M.; RAMALHO, J.C. Combined effects of elevated $\left[\mathrm{CO}_{2}\right]$ and high temperature on leaf mineral balance in Coffea spp. plants. Climatic Change, v.126, p.365-379, 2014. DOI: 10.1007/s10584-014-1236-7.

NORTON, R.; FITZGERALD, G.; TAUSZ, M. Climate change and wheat crop responses - FACEing the future. Better Crops, v.95, p.12-13, 2011.

PAVINATO, P.S.; ROSOLEM, C.A. Disponibilidade de nutrientes no solo: decomposição e liberação de compostos orgânicos de resíduos vegetais. Revista Brasileira de Ciência do Solo, v.32, p.911-920, 2008. DOI: 10.1590/ S0100-06832008000300001.

POORTER, H.; NAVAS, M.-L. Plant growth and competition at elevated $\mathrm{CO}_{2}$ : winners, losers and functional groups. New Phytologist, v.157, p.175-198, 2003. DOI: 10.1046/j.1469-81 37.2003.00680.x.

RAIJ, B. van; ANDRADE, J.C. de; CANTARELLA, H.; QUAGGIO, J.A. (Ed.). Análise química para avaliação da fertilidade de solos tropicais. Campinas: IAC, 2001. 285p.

RAIJ, B. van; CANTARELLA, H.; QUAGGIO, J.A.; FURLANI, A.M.C. (Ed.). Recomendações de adubação e calagem para o estado de São Paulo. 2.ed. Campinas: IAC, 1997. 285p. (IAC. Boletim técnico, 100).

RAMALHO, J.C.; RODRIGUES, A.P.; SEMEDO, J.N.; PAIS, I.P.; MARTINS, L.D.; SIMÕES-COSTA, M.C.; LEITÃO, A.E.; FORTUNATO, A.S.; BATISTA-SANTOS, P.; PALOS, I.M.; TOMAZ, M.A.; SCOTTI-CAMPOS, P.; LIDON, F.C.; DAMATTA, F.M. Sustained photosynthetic performance of Coffea spp. under long-term enhanced $\left[\mathrm{CO}_{2}\right]$. PLoS ONE, v.8, article ID e82712, 2013. DOI: 10.1371/journal.pone.0082712.

ROSOLEM, C.A.; VICENTINI, J.P.T.M.M.; STEINER, F. Suprimento de potássio em função da adubação potássica residual em um Latossolo Vermelho do Cerrado. Revista Brasileira de Ciência do Solo, v.36, p.1507-1515, 2012. DOI: 10.1590/S0100-06832012000500015.

SUJATHA, K.B.; UPRETY, D.C.; RAO, D.N.; RAO, P.R.; DWIVEDI, N. Up-regulation of photosynthesis and sucrose-P synthase in rice under elevated carbon dioxide and temperature conditions. Plant, Soil and Environment, v.54, p.155-162, 2008.

Pesq. agropec. bras., Brasília, v.50, n.11, p.1087-1096, nov. 2015 DOI: 10.1590/S0100-204X2015001100012 
TEDESCO, M.J.; GIANELLO, C.; BISSANI, C.A.; BOHNEN, H.; VOLKWEISS, S.J. Análises de solo, plantas e outros materiais. 2.ed. Porto Alegre: UFRGS, Departamento de Solos, 1995. 174p. (UFRGS. Boletim técnico, 5).

VALARINI, V.; BATAGLIA, O.C.; FAZUOLI, L.C. Macronutrientes em folhas e frutos de cultivares de café arábica de porte baixo. Bragantia, v.64, p.661-672, 2005. DOI: 10.1590/ S0006-87052005000400016.
WALTER, L.C.; ROSA, H.T.; STRECK, N.A. Mecanismos de aclimatação das plantas à elevada concentração de $\mathrm{CO}_{2}$. Ciência Rural, v.45, p.1564-1571, 2015. DOI: 10.1590/0103-8478cr20140527.

YAN, F.; SCHUBERT, S.; MENGEL, K. Soil pH increase due to biological decarboxylation of organic anions. Soil Biology and Biochemistry, v.28, p.617-624, 1996. DOI: 10.1016/0038-0717(95)00180-8.

$\overline{\text { Recebido em } 18 \text { de maio de } 2015 \text { e aprovado em } 6 \text { de outubro de } 2015}$ 